\section{PSQ-107 ACTIVE PHARMACOVIGILANCE AND DEDICATED PHARMACIST: THE EXPERIENCE OF A TERRITORIAL HEALTHCARE HOSPITAL}

${ }^{1}$ A Pirrone ${ }^{*},{ }^{2} \mathrm{~A}$ Spagnuolo, ${ }^{2} \mathrm{G}$ Caravella, ${ }^{1} \mathrm{C}$ Bertino, ${ }^{1} \mathrm{E}$ Della Fontana, ${ }^{1} \mathrm{M}$ Di Gerardo,

${ }^{2} \mathrm{R}$ Cursano. 'Universita Degli Studi Di Milano, Scienze Farmaceutiche-Scuola Di Specializzazione in Farmacia Ospedaliera, Milano, Italy; ${ }^{2}$ Asst Melegnano Martesana, Hospital Pharmacy, Vizzolo Predabissi, Italy

\subsection{6/ejhpharm-2020-eahpconf.424}

Background and importance The main objectives of active pharmacovigilance (FA) projects are to identify potential warning signs regarding the use of drugs, prevent adverse reactions (ADRs) and promote the safe and effective use of medicines. Aim and objectives The aim of this work was to assess the introduction of measures designed to tackle clinical appropriateness and safety, organisation of interdisciplinary work groups, awareness of health personnel and monitoring adherence to chronic therapies.

Material and methods Analysis of reports included in a national pharmacovigilance network (RNF) by our territorial healthcare hospital (ASST) was conducted, during two time periods of 230 days each, one preceding and one following the beginning of the FA project with a dedicated pharmacist.

Results In the first period, 66 reports were included, of which 15 were serious and 51 were not serious. The signallers were physicians (55) and pharmacists (11). The age group most involved were those aged $>65$ year (50\%). Women were involved in $70 \%$ of ADRs. There were 76 suspected drugs, $50 \%$ represented by antineoplastics. The most reported ADRs, described for preferred term (PT), were erythema (8), pruritus (7), hypotension (6) and urticaria (5), for a total of 134 different PTs. In the second period there were 107 reports, of which 79 were not serious and 28 were serious (2 deaths). In addition to pharmacists (66) and physicians (36), 5 ADRs were added by other health professionals. The age group most involved was 18-65 years (56\%). There were 134 suspected drugs, with a $106 \%$ increase in reports of suspected non-antineoplastic drugs (30-62). The most reported adverse reactions were pruritus, dyspnoea, erythema and neutropenia, for a total of 241 PTs (80\% increase compared with the first period).

Conclusion and relevance In addition to a significant increase in the number of reports, there was an evident increase in the type of drugs and reactions reported, thanks to the collaboration and awareness of health personnel and patients. With the peculiar organisation of the ASST, divided into two sectors (namely territorial network and hospital centre), the pharmacist can carry out pharmacovigilance activity on various levels, such as hospital department, direct distribution of drugs, family counselling, vaccination centres and assistance continuity services. This promotes the increase in quantity and quality of reports, and contributes to improving and updating the safety profile of drugs, favouring the appropriateness of their use.

\section{REFERENCES AND/OR ACKNOWLEDGEMENTS}

No conflict of interest.

\section{PSQ-108 INFECTION WITH CLOSTRIDIUM DIFFICILE: RISK FACTORS AND PHARMACOTHERAPEUTIC MANAGEMENT}

MDV Sanchez Matamoros Piazza, V Vazquez Vela, C Mora Herrera, C Puivecino Moreno*, A Varas Perez. Hospital Universitario Jerez De La Frontera, Pharmacy Senvices, 41007, Spain

\subsection{6/ejhpharm-2020-eahpconf.425}

Background and importance The incidence and severity of cases of Clostridium difficile infection (CDI) has been increasing, as well as hospital stays and hospitalisation costs.

Aim and objectives To analyse the therapeutic approach of CDI and the contribution of risk factors.

Material and methods A retrospective observational study was conducted in patients with culture and/or positive toxins for CD during 2018. The information was obtained after review of the clinical histories of patients with CDI: demographic data, previous antibiotic treatment, risk factors (age, SNG, treatment with immunosuppressants, PPIs, laxatives, NSAIDs or IBD), severity of the episode, treatment established, subsequent recurrences $(<4$ months after treatment $)$ and complications.

Results Thirty patients were included, $75.86 \%$ with a positive toxin. Average age was 64.12 years, $65.52 \%$ of patients were women and $96.55 \%$ had received previous antibiotic treatments (broad spectrum penicillins, cephalosporins and quinolones). Risk factors: $58.62 \%$ (17) >65 years, $82.76 \%$ (24) undergoing treatment with PPIs, 20.68\% (6) NSAIDs, $13.79 \%$ (4) laxatives, 31.03\% (9) immunosuppressants, $13.79 \%$ (4) had IBD and $10.34 \%$ (3) SNG.

CDI were considered mild-moderate in $93.1 \%$ (28) of patients and severe in $6.9 \%$ (2). They were treated with vancomycin (68.97\%), metronidazole (6.9\%) and vancomycin/metronidazole (17.24\%), and 2 were untreated. Most common pattern was vancomycin $250 \mathrm{mg} / 6$ hours for an average of 14 days. One patient presented with toxicoderma after vancomycin and 10.3\% (3) presented recurrences (0.5-3.5 months): 2 patients were treated with vancomycin/metronidazole, and after a new recurrence with vancomycin/fidaxomycin. In the other patient, bezlotuxumab/vancomycin was used first with a good resolution.

In $79.31 \%$ (23) of patients their infectious condition was resolved satisfactorily. Three patients presented complications (two sepsis and one pseudomembranous colitis). The rest of the follow-up losses were due to transfer (3) and success for other reasons (1).

Conclusion and relevance The majority of patients presented with mild-moderate CDI and vancomycin was used as usual therapy with good resolution. Risk factors included prior use of antibiotics, immunosuppressants and PPIs. It is essential to stratify patients according to severity and re-treat according to the previous episode.

\section{REFERENCES AND/OR ACKNOWLEDGEMENTS}

No conflict of interest. 